\title{
The EUV Flux of Chromospherically Active Binary Stars
}

\author{
ALVARO GIMÉNEZ ${ }^{1}$ AND CONSTANZE LA DOUS ${ }^{2}$ \\ ${ }^{1}$ LAEFF, INTA, Villafranca del Castillo, Apartado 50727, 28080 Madrid, Spain \\ ${ }^{2}$ IUE Observatory, ESA, Villafranca del Castillo, Apartado 50727, 28080 Madrid, Spain
}

We have compared EUVE and ROSAT/WFC photometric measurements of chromospherically active binary systems at around $100 \AA$. Long term variations have found for some $20 \%$ of the systems as well as a tight linear correlation between the EUV and soft X-ray fluxes.

The recent all-sky survey of the EUVE satellite has permitted a study of the emission flux in this spectral range for chromospherically active late-type stars. These binaries are among the most numerous objects observed by $E U V E$ due to the fact that they show the brightest coronae among late-type, relatively nearby, stars.

The EUV emission from active binaries originates in a hot plasma in their coronae. They are known to have two temperature components: one around 20 million $\mathrm{K}$ (similar to solar flares plasma) and a cooler one of some 5 million $\mathrm{K}$. The bulk of the emission is concentrated in lines of highly ionized species of elements like iron and silicon. In principle, by measuring the relative strengths of the coronal emission lines, the densities and temperatures of the plasma could be inferred. But a detailed plasma diagnostic is only possible through high resolution spectra which are difficult to obtain except for the very brightest systems.

We have performed a comparison of photometric data in the EUV range of chromospherically active binaries, looking for long term variability. For this purpose, we have taken the EUVE Source Catalog published by Bowyer et al. (1994), which contains a total of 410 objects and the ROSAT WFC Bright Source Catalogue by Pounds et al. (1993), which includes 383 sources. Only 46 of the identified objects by $E U V E$ are also included in the Catalog of Chromospherically Active Binary Stars by Strassmeier et al. (1993), which contains 206 systems in total. As expected, only those binaries with a high level of coronal activity and at short distances were detected. Four sources were not detected with the Wide Field Camera (WFC) of ROSAT (AG Dor, DK Dra, 42 Cap and HD 57853) while EUVE did not observe 6 WFC sources included in Strassmeier et al. (1993) catalogue ( $\zeta$ And, V1285 Aql, OU Gem, DQ Leo, TW Lep and AR Psc). The Second EUVE Source Catalog (Bowyer et al. 1996) became available to us too late to be fully used in this comparison. Nevertheless, a preliminary evaluation led us to the same overall conclusions, with a number of detected active binaries increased to 53 and only 2 systems from the WFC catalogue not detected by $E U V E$.

Long term variability in the EUV range was checked by comparing the obtained fluxes with the EUVE satellite during 1992 and by the WFC during 1990. Short term variations, like flares, are not considered in the tabulated input data. 42 systems were observed by the two satellites and 6 of them did show relatively large differences in flux: mainly, UX Ari and $\alpha$ Aur, but also VY Ari, BH CVn, II Peg and AR Lac. All of them are well known for their conspicuous levels of activity and variability in other wavelength ranges. Differences are not so obvious in less active systems and, when instrumental corrections are taken into account, only 4 more binaries were found to have flux differences larger than $50 \%$ of the average value (UX For, V815 Her, BY Dra and ER Vul). This indicates that some 15 to $25 \%$ of the sample show large variations, in good agreement with similar 
results in the far UV and soft X-ray ranges. Figure 1 shows the comparison of $E U V E$ and WFC fluxes with indication of the variable sources.

Taking out the variable systems, a good linear relation is found between the EUVE (Lexan/B) and WFC(S1) fluxes, or instrumental correction, that can be expressed in terms of counts $\mathrm{s}^{-1}$ as,

$$
E U V E=-0.012( \pm 0.007)+2.14( \pm 0.07) \mathrm{WFC}
$$

To check the soft X-ray variability, we have compared the fluxes measured by the PSPC on board ROSAT (Dempsey et al. 1993) and the IPC of the Einstein observatory (Schmitt et al. 1990). Only 14 binaries of our sample of active binaries were observed by the two instruments and 4 of them were found to be variable, actually the same systems detected in the EUV (UX Ari, BH CVn, II Peg, and VY Ari) within the subset. Finally, we have related the EUV flux in the EUVE Lexan/B band with the PSPC measurements for the non-variable binaries in common to both catalogues (24 systems) and, again, a good linear relation was found though the position of $\sigma \mathrm{CrB}$ may show an anomalous behaviour with a too high EUV flux.

\section{REFERENCES}

BOWYER, S. ET AL. 1994, ApJS, 93, 569

BOWYER, S. ET AL. 1996, ApJS, in press

DEMPSEY, R. C. ET AL. 1993, ApJS, 86, 599

Pounds, K. A. ET AL. 1993, MNRAS, 260, 77

SchMitT, J. H. M. M. ET AL. 1990, ApJ, 365, 704

Strassmeier, K. G. ET AL. 1993, A\&AS, 100, 173 